Vietnam Journal of Mechanics, VAST, Vol.41, No.3 (2019), pp. $257-272$

DOI: https://doi.org/10.15625/0866-7136/13723

\title{
VIBROACOUSTIC ANALYSIS OF A CLAMPED FINITE ORTHOTROPIC LAMINATED DOUBLE-COMPOSITE PLATE WITH AN AIR CAVITY
}

\author{
Tran Ich Thinh ${ }^{1, *}$, Pham Ngoc Thanh ${ }^{2}$ \\ ${ }^{1}$ Hanoi University of Science and Technology, Vietnam \\ ${ }^{2}$ Viet Tri University of Industry, Phu Tho, Vietnam \\ *E-mail: thinh.tranich@.hust.edu.vn
}

Received: 02 April 2019 / Published online: 20 August 2019

\begin{abstract}
The vibroacoustic analysis of a clamped finite orthotropic laminated doublecomposite plate with a closed air cavity is investigated analytically based on the classical laminated plate theory. Using the method of modal decomposition, a double Fourier series solution is obtained to characterize the vibroacoustic performance of the structure. The sound transmission loss (STL) is calculated from the ratio of incident to transmitted acoustic powers. The accuracy of the solution is shown with comparing the STL values obtained from this presented model with the experimental and theoretical results available in literature. The soundproof ability of finite double-composite plate with a clamed boundary is shown. The effects of thickness of faceplates, thickness of air cavity and the angles of the incident sound are systematically examined.
\end{abstract}

Keywords: vibroacoustic analysis; double-composite plate; sound transmission loss.

\section{INTRODUCTION}

Double-plate with enclosed air cavity is widely used in modern vehicle and building industry, aerospace and aeronautical structures, etc. due to superior sound insulation ability compared to single plate. In order to estimate the sound insulation of a doubleplate, the determination of sound transmission loss (STL) across this structure at different frequencies is necessary.

Many literatures deal with theoretical, experimental, statistical and numerical models to determine the STL of single or double-plates [1-5]. Statistical energy analysis (SEA) is a modeling procedure which uses energy flow relationships for the theoretical estimation of the vibration response levels and the noise radiation from structures. Maidanik [6] has developed this method to analyze the vibration response of a complex structure under force or sound excitation. Using finite element method (FEM), Ruzzene [7] evaluated the acoustic characteristics and sound transmission reduction index of sandwich beams with honeycomb truss core in terms of structural response for low and high frequencies.

(C) 2019 Vietnam Academy of Science and Technology 
In general, FEM is applied together with BEM (boundary element method) to deal with the boundary and interface conditions. Although FEM and BEM are much effective for low frequencies but require high computational cost for high frequencies. While the SEA method is less effective at relatively low frequencies, but it is substantially effective in providing sound transmission estimates for complex structures at high frequencies. The first experimental test on the sound insulation properties of double-panel structures was carried out by London [8]. The same author studied, both theoretically and experimentally, the transmission loss of a panel constituted by two aluminium faces and plywood core subjected to a reverberant field. Later, Carneal and Fuller [1] presented an analytical and experimental study of active structural acoustic control of noise transmission through double panel systems. The experimental measurements in [1] were carried out on clamped aluminum double-plate, whereas the theoretical model was constructed on simply supported boundary condition. In order to experimentally verify the model, the stiffness of the simply supported double-plate was increased artificially by a factor of $\sqrt{2}$ for each boundary to approximate the clamped boundary condition. Experimental tests are presented as well, showing the increasing of the transmission loss of the double wall with the increasing of the air gap. Similarly Kropp and Rebillard [9] and Villot et al. [10] studied the air-borne sound insulation capability of infinite double wall constructions. Recently, the work of Chazot and Guyader [11] has presented a prediction of sound transmission loss through finite double panels by using a patch-mobility method. This method and FEM give the same results when comparing transmission loss of double aluminum plates. In addition, to improve the sound isolation properties of double-panel partitions, various active control strategies have been proposed, both experimentally and theoretically which are significant from the viewpoint of practical noise control by Bao and Pan [12].

The double-plates filled with absorbent materials are commonly used in many systems for vibration damping and noise control. The transmission loss of sound across these sandwich structures has been extensively investigated. For example, Brouard et al. [13] proposed a general method to model sound propagation in layered systems such as fluid-saturated porous layers while Lauriks et al. [14] developed a transfer matrix model to study the transmission loss through panels with solid porous layers, with the Biot model employed to describe sound propagation through the porous material. In [15] a finite element formulation for sound transmission through double wall sandwich panels with viscoelastic core is presented by Larbi et al. Panneton et al. [16] proposed a three-dimensional (3D) finite element model to calculate the loss of sound transmission through multilayer structures containing porous absorbent materials. The structures considered vary according to whether the filling porous material is bonded or not to the faceplates. Making use of twodimensional (2D) elasticity theory, Chonan and Kugo [17] addressed a model to examine the sound transmission characteristics of a three-layered panel excited by plane waves. Kang et al. [18] used the method of Gauss distribution function for incident energy to investigate the STL of multilayered panels such as doubleplate structures embedded with porous materials. Bolton et al. [19] calculated random incidence transmission loss through double-leaf panels lined with elastic porous materials. 
More recently, the problem of sound transmission across double-plate structures of finite extent was solved by considering simple boundary conditions $[5,11,20]$ on the basis of modal superposition theory from different viewpoints. Lu and Xin [5] presented an analytical approach to investigate the sound transmission across rectangular metallic (isotropic) double-panel structure containing an air cavity under various boundary constraints by using the method of modal function and the weighted residual (Galerkin) method. Numerous analytical investigations on the STL through a finite isotropic doubleplate have been performed $[1,5,13,14]$. However, to the author's best knowledge, there exist a few of studies on the vibroacoustic behavior and STL across a finite orthotropic clamped single and double-composite plate. More recently, Thinh and Thanh calculated the loss of sound propagation through a finite simply supported [21] and clamped [22] composite plate and also considered the effect of some parameters on the sound insulation ability of the plate.

The objective of this study is to develop a model to describe accurately the vibroacoustic performance of a clamped finite orthotropic laminated double-composite plate filled with air. In this investigation, the theoretical and existing experimental results were compared and reached a good agreement. The influence of several key system parameters (e.g., thickness of faceplate, the thickness of air cavity and the sound incident angles) on sound transmission loss is considered.

\section{THEORETICAL FORMULATION}

\subsection{Description of the composite double-plate}

A finite double-plate with air cavity consists of two thin rectangular plates made of orthotropic laminated composite, as shown in Fig. 1. The double-plate is fully clamped along their edges to an infinite large acoustic rigid baffle. The two single composite plates have similar geometric parameters and mechanical properties. The bottom and upper plates have thicknesses $h$ and are separated by an air cavity of thickness $H$. The doubleplate divides the spatial space into two fields, i.e., sound incidence field $(z<0)$ and sound transmitting field $(z>H+2 h)$. The incident elevation and azimuth angles are denoted by $\varphi$ and $\theta$, respectively (Fig. 1).

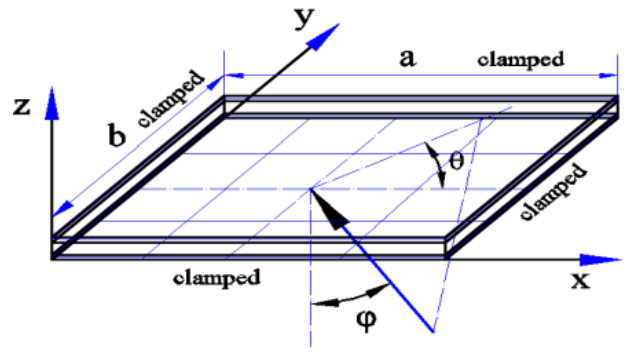

(a) Global view

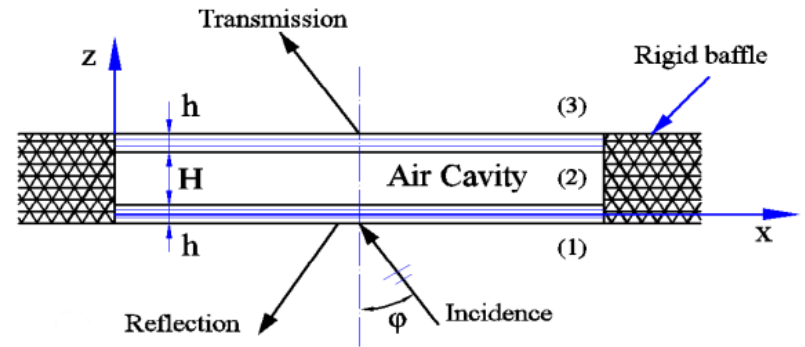

(b) Side view

Fig. 1. Schematic of a clamped composite double-plate 


\subsection{Formulation of the vibroacoustic problem}

The vibroacoustic response of an orthotropic symmetric double-composite plate with air cavity (Fig. 1) induced by sound excitation is governed by [22-24]

$$
\begin{aligned}
D_{11} \frac{\partial^{4} w_{1}(x, y ; t)}{\partial x^{4}} & +2\left(D_{12}+2 D_{66}\right) \frac{\partial^{4} w_{1}(x, y ; t)}{\partial x^{2} \partial y^{2}}+D_{22} \frac{\partial^{4} w_{1}(x, y ; t)}{\partial y^{4}} \\
& +m^{*} \frac{\partial^{2} w_{1}(x, y ; t)}{\partial^{2} t}-j \omega \rho_{0}\left[\Phi_{1}(x, y, z ; t)-\Phi_{2}(x, y, z ; t)\right]=0, \\
D_{11} \frac{\partial^{4} w_{2}(x, y ; t)}{\partial x^{4}}+ & 2\left(D_{12}+2 D_{66}\right) \frac{\partial^{4} w_{2}(x, y ; t)}{\partial x^{2} \partial y^{2}}+D_{22} \frac{\partial^{4} w_{2}(x, y ; t)}{\partial y^{4}} \\
& +m^{*} \frac{\partial^{2} w_{2}(x, y ; t)}{\partial^{2} t}-j \omega \rho_{0}\left[\Phi_{2}(x, y, z ; t)-\Phi_{3}(x, y, z ; t)\right]=0,
\end{aligned}
$$

where $w_{1,2}$ are the transverse displacements of the two single-plates; $D_{i j}(i j=11,12,22,66)$ are the flexural rigidities; $m^{*}$ is the surface density of the plate; $\rho_{0}$ is the air density; $j=\sqrt{-1}, \omega$ is the angular frequency of the incident sound and $\Phi_{i}(i=1,2,3)$ are the velocity potentials of the three acoustic fields: sound incidence field, air cavity field and transmitting field, respectively.

The flexural rigidity of laminated composite plate is determined by (see any textbook of mechanics of composite materials)

$$
D_{i j}=\frac{1}{3} \sum_{k=1}^{n} Q_{i j}^{k}\left(z_{k+1}^{3}-z_{k}^{3}\right),
$$

where $Q_{i j}$ the reduced stiffnesses of the $k^{\text {th }}$ layer are defined as

$$
Q_{11}=\frac{E_{1}}{1-v_{12} v_{21}} ; Q_{12}=\frac{v_{12} E_{1}}{1-v_{12} v_{21}} ; Q_{22}=\frac{E_{2}}{1-v_{12} v_{21}} ; Q_{66}=G_{12} ; \frac{v_{12}}{E_{1}}=\frac{v_{21}}{E_{2}},
$$

and $E_{1}, E_{2}, G_{12}, v_{12}$ are the $k^{\text {th }}$ layer elastic constants. Note that, for an isotropic plate, $D_{11}=D_{22}=E h^{3} /\left[12\left(1-v^{2}\right)\right], D_{12}=v D_{11}, D_{66}=G h^{3} / 12$.

The displacements of the two composite plate induced by a harmonic sound wave can be expressed as

$$
w_{1}(x, y ; t)=w_{01} e^{-j\left(k_{x} x+k_{y} y-\omega t\right)} ; w_{2}(x, y ; t)=w_{02} e^{-j\left(k_{x} x+k_{y} y-\omega t\right)} .
$$

The acoustic velocity potential in the incident field (field (1) on Fig. 1) is defined by the following sum $[5,14,20]$

$$
\Phi_{1}(x, y, z ; t)=I e^{-j\left(k_{x} x+k_{y} y+k_{z} z-\omega t\right)}+\beta e^{-j\left(k_{x} x+k_{y} y-k_{z} z-\omega t\right)} .
$$

The first term is the velocity potential of the incident sound wave, the second term represents the reflected plus radiated waves. $I$ and $\beta$ in (6) are the amplitudes of the incident positive-going wave and the reflected plus radiated negative-going waves, respectively.

Similarly, the acoustic velocity potential in the air cavity (field (2) on Fig. 1) can be written as

$$
\Phi_{2}(x, y, z ; t)=\varepsilon e^{-j\left(k_{x} x+k_{y} y+k_{z} z-\omega t\right)}+\psi e^{-j\left(k_{x} x+k_{y} y-k_{z} z-\omega t\right)},
$$


where $\varepsilon$ and $\psi$ are the amplitudes of positive-going wave and negative-going waves of field (2), respectively. In the transmitting field (field (3) on Fig. 1), there is only a transmitted wave with the amplitude $\gamma$ and its velocity potential is given by

$$
\Phi_{3}(x, y, z ; t)=\gamma e^{-j\left(k_{x} x+k_{y} y+k_{z} z-\omega t\right)} .
$$

These wave numbers are determined by the incident angle $\varphi$ and azimuth angle $\theta$ of the incident sound wave as

$$
k_{x}=k_{0} \sin \varphi \cos \theta ; \quad k_{y}=k_{0} \sin \varphi \sin \theta ; \quad k_{z}=k_{0} \cos \varphi,
$$

where $k_{0}=\omega / c_{0}$ is the acoustic wave number in air and $c_{0}$ is the acoustic speed in the air.

The boundary conditions for the fully clamped double-plate can be expressed as

$$
\begin{aligned}
& \text { at } x=0 \text { and } a, w_{1}=w_{2}=0, \frac{\partial w_{1}}{\partial x}=\frac{\partial w_{2}}{\partial x}=0, \\
& \text { at } y=0 \text { and } b, w_{1}=w_{2}=0, \frac{\partial w_{1}}{\partial y}=\frac{\partial w_{2}}{\partial y}=0 .
\end{aligned}
$$

At the air-plate interface, the following velocity compatibility equations must be satisfied $[24,25]$

$$
-\left.\frac{\partial \Phi_{1}}{\partial z}\right|_{z=0}=j \omega w_{1} ;-\left.\frac{\partial \Phi_{2}}{\partial z}\right|_{z=h}=j \omega w_{1} ;-\left.\frac{\partial \Phi_{2}}{\partial z}\right|_{z=H+h}=j \omega w_{2} ;-\left.\frac{\partial \Phi_{3}}{\partial z}\right|_{z=H+2 h}=j \omega w_{2} .
$$

Under the excitation of the harmonic sound waves, the flexural motions of the bottom and upper plates can be expressed in the form of double series as

$$
w_{1}(x, y ; t)=\sum_{m=1}^{\infty} \sum_{n=1}^{\infty} \varphi_{m n}(x, y) X_{1, m n}(t) ; \quad w_{2}(x, y ; t)=\sum_{m=1}^{\infty} \sum_{n=1}^{\infty} \varphi_{m n}(x, y) X_{2, m n}(t),
$$

where the modal function $\varphi_{m n}$ and modal coefficients $X_{i, m n}$ and $X_{i, m n}$ are defined by [26]

$$
\begin{aligned}
\varphi_{m n}(x, y) & =\left(1-\cos \frac{2 m \pi x}{a}\right)\left(1-\cos \frac{2 n \pi x}{b}\right), \\
X_{1, m n}(t) & =A_{1, m n} e^{j \omega t}, \quad X_{2, m n}(t)=A_{2, m n}, e^{j \omega t}
\end{aligned}
$$

where $A_{1, m n}, A_{2, m n}$ are the modal coefficients of the bottom and upper plates, respectively and will be determined in the following subsection.

Thus, the velocity potentials for the sound incident field, air cavity, and sound transmitting field may be expressed as

$$
\begin{aligned}
\Phi_{1}(x, y, z ; t)= & \sum_{m=1}^{\infty} \sum_{n=1}^{\infty} I_{m n} \varphi_{m n} e^{-j\left(k_{z} z-\omega t\right)}+\sum_{m=1}^{\infty} \sum_{n=1}^{\infty} \beta_{m n} \varphi_{m n} e^{-j\left(-k_{z} z-\omega t\right)}, \\
\Phi_{2}(x, y, z ; t)= & \sum_{m=1}^{\infty} \sum_{n=1}^{\infty} \varepsilon_{m n} \varphi_{m n} e^{-j\left(k_{z} z-\omega t\right)}+\sum_{m=1}^{\infty} \sum_{n=1}^{\infty} \psi_{m n} \varphi_{m n} e^{-j\left(-k_{z} z-\omega t\right)}, \\
& \Phi_{3}(x, y, z ; t)=\sum_{m=1}^{\infty} \sum_{n=1}^{\infty} \gamma_{m n} \varphi_{m n} e^{-j\left(k_{z} z-\omega t\right)},
\end{aligned}
$$


where the coefficients $I_{m n}, \beta_{m n}, \varepsilon_{m n}, \psi_{m n}$ and $\gamma_{m n}$ are determined by

$$
\chi_{m n}=\frac{4}{a b} \int_{0}^{b} \int_{0}^{a} x e^{-j\left(k_{x} x+k_{y} y\right)} \cos \frac{2 m \pi x}{a} \cos \frac{2 n \pi x}{b} d x d y,
$$

where the symbol $\chi$ can be referred to any of the coefficients $I, \beta, \varepsilon, \psi$ and $\gamma$.

\subsection{Determination of the modal coefficients}

Let $\xi_{1}, \xi_{2}$ and $\xi_{3}$ represent the acoustic particle displacements in the incident, the air cavity and the transmitted air medium, respectively. The air particle displacements and the acoustic pressures are related by the equation, as [27]

$$
\begin{aligned}
& \frac{\partial^{2}}{\partial t^{2}} \xi_{1}=-\left.\frac{1}{\rho_{0}} \frac{\partial p_{1}}{\partial z}\right|_{z=0} ; \quad \frac{\partial^{2}}{\partial t^{2}} \xi_{2}=-\left.\frac{1}{\rho_{0}} \frac{\partial p_{2}}{\partial z}\right|_{z=h} ; \\
& \frac{\partial^{2}}{\partial t^{2}} \xi_{2}=-\left.\frac{1}{\rho_{0}} \frac{\partial p_{2}}{\partial z}\right|_{z=H+h} ; \quad \frac{\partial^{2}}{\partial t^{2}} \xi_{3}=-\left.\frac{1}{\rho_{0}} \frac{\partial p_{3}}{\partial z}\right|_{z=H+2 h},
\end{aligned}
$$

where the acoustic pressure can be expressed by the acoustical velocity potentials through Bernoulli's equation, as [24,25]

$$
p_{i}=\rho_{0}\left[\frac{\partial \Phi_{i}}{\partial t}\right], \quad(i=1,2,3) .
$$

One can express the displacements of the air particle adjacent to the plate in each region as

$$
\xi_{1}=\xi_{10} e^{-j\left(k_{x} x+k_{y} y-\omega t\right)} ; \xi_{2}=\xi_{20} e^{-j\left(k_{x} x+k_{y} y-\omega t\right)} ; \xi_{3}=\xi_{30} e^{-j\left(k_{x} x+k_{y} y-\omega t\right)} .
$$

Substituting (20)-(21) into (19), and using the acoustical velocity potentials (6)-(8), one can obtain

$$
\begin{gathered}
\xi_{10}=\left(\sum_{m=1}^{\infty} \sum_{n=1}^{\infty} I_{m n} \varphi_{m n}-\sum_{m=1}^{\infty} \sum_{n=1}^{\infty} \beta_{m n} \varphi_{m n}\right) \frac{k_{z}}{\omega} e^{j\left(k_{x} x+k_{y} y\right)}, \\
\xi_{20}=\left(\sum_{m=1}^{\infty} \sum_{n=1}^{\infty} \varepsilon_{m n} \varphi_{m n}-\sum_{m=1}^{\infty} \sum_{n=1}^{\infty} \psi_{m n} \varphi_{m n}\right) \frac{k_{z}}{\omega} e^{j\left(k_{x} x+k_{y} y\right)}, \\
\xi_{30}=\sum_{m=1}^{\infty} \sum_{n=1}^{\infty} \gamma_{m n} \varphi_{m n} \frac{k_{z}}{\omega} e^{j\left(k_{x} x+k_{y} y\right)},
\end{gathered}
$$

As well, continuity conditions at air-composite plate interfaces require that the displacements of the air particles adjacent to the plate and those of the attached plate particles should be equal. Therefore, the displacement continuity condition can be written as

$$
\left.\xi_{10}\right|_{z=0}=w_{01}=\left.\xi_{20}\right|_{z=h} ;\left.\quad \xi_{20}\right|_{z=H+h}=w_{02}=\left.\xi_{20}\right|_{z=H+2 h} .
$$

Substituting Eqs. (15)-(17) into Eq. (11) one can obtain

$$
\beta_{m n}=I_{m n}-\frac{\omega}{k_{z}} A_{1, m n} ; \quad \varepsilon_{m n}=\frac{\omega\left(A_{1, m n} e^{j k_{z} H}-A_{2, m n}\right)}{k_{z}\left(e^{-j k_{z}(h-H)}-e^{-j k_{z}(H+h)}\right)} .
$$




$$
\psi_{m n}=\frac{\omega\left(A_{2, m n}-A_{1, m n} e^{-j k_{z} H}\right)}{k_{z}\left(e^{j k_{z}(h-H)}-e^{j k_{z}(H+h)}\right)} ; \quad \gamma_{m n}=\frac{\omega A_{2, m n} e^{j k_{z}(H+2 h)}}{k_{z}} .
$$

Substituting Eqs. (12) and (25)-(26) into Eqs. (1)-(2) and applying the orthogonal properties of modal functions, one gets

$$
\begin{gathered}
\ddot{X}_{1, m n}(t)+\omega_{1, m n}^{2} X_{1, m n}(t)-\frac{j \omega \rho_{0}}{m^{*}}\left[\left(I_{m n}-\varepsilon_{m n}\right) e^{-j\left(k_{z} z-\omega t\right)}+\left(\beta_{m n}-\psi_{m n}\right) e^{-j\left(-k_{z} z-\omega t\right)}\right]=0, \\
\ddot{X}_{2, m n}(t)+\omega_{2, m n}^{2} X_{2, m n}(t)-\frac{j \omega \rho_{0}}{m^{*}}\left[\left(\varepsilon_{m n}-\gamma_{m n}\right) e^{-j\left(k_{z} z-\omega t\right)}+\psi_{m n} e^{-j\left(-k_{z} z-\omega t\right)}\right]=0 .
\end{gathered}
$$

The natural frequencies of the bottom and upper orthotropic laminated composite plates are determined by [22]

$$
\omega_{i, m n}^{2}=\frac{\iint_{A}\left(D_{11} \frac{\partial^{4} \varphi_{m n}}{\partial^{4} x}+2\left(D_{12}+2 D_{66}\right) \frac{\partial^{4} \varphi_{m n}}{\partial^{2} x \partial^{2} y}+D_{22} \frac{\partial^{4} \varphi_{m n}}{\partial^{4} y}\right) \varphi_{m n} d A}{m^{*} \iint_{A} \varphi_{m n} \varphi_{m n} d A}, \quad(i=1,2) .
$$

Using Eq. (14), Eqs. (27) and (28) can be expressed in matrix form as

$$
\left[\begin{array}{ll}
R_{11} & R_{12} \\
R_{21} & R_{22}
\end{array}\right]\left\{\begin{array}{l}
A_{1, m n} \\
A_{2, m n}
\end{array}\right\}=\left\{\begin{array}{l}
S \\
0
\end{array}\right\}
$$

where

$$
\begin{aligned}
& R_{11}=\omega_{1, m n}^{2}-\omega^{2}-\frac{j \omega^{2} \rho_{0}}{k_{z} m^{*}}\left[\frac{e^{j k_{z} H}}{e^{-j k_{z}(h-H)}-e^{-j k_{z}(H+h)}}-\frac{e^{-j k_{z} H}}{e^{j k_{z}(h-H)}-e^{j k_{z}(H+h)}}\right], \\
& R_{12}=\frac{j \omega^{2} \rho_{0}}{k_{z} m^{*}}\left[\frac{1}{e^{-j k_{z}(h-H)}-e^{-j k_{z}(H+h)}}-\frac{1}{e^{j k_{z}(h-H)}-e^{j k_{z}(H+h)}}\right], \\
& R_{21}=\frac{j \omega^{2} \rho_{0}}{k_{z} m^{*}}\left[\frac{e^{j k_{z} H}}{e^{-j k_{z}(h-H)}-e^{-j k_{z}(H+h)}}-\frac{e^{-j k_{z} H}}{e^{j k_{z}(h-H)}-e^{j k_{z}(H+h)}}\right], \\
& R_{22}=\omega_{2, m n}^{2}-\omega^{2}-\frac{j \omega^{2} \rho_{0}}{k_{z} m^{*}}\left[\frac{1}{e^{j k_{z}(h-H)}-e^{j k_{z}(H+h)}}-\frac{1}{e^{-j k_{z}(h-H)}-e^{-j k_{z}(H+h)}}+e^{j k_{z}(H+2 h)}\right] \text {, } \\
& S=\frac{2 j \omega \rho_{0} I_{m n}}{m^{*}} .
\end{aligned}
$$

After solving the system of equations (30), the unknown coefficients $A_{1, m n}$ and $A_{2, m n}$ are determined, all the other quantities such as $w_{1}, w_{2}$ and the coefficients $\left(\beta_{m n}, \varepsilon_{m n}, \psi_{m n}\right.$ and $\left.\gamma_{m n}\right)$ are also determined. Thence, the analysis of sound transmission across a doublecomposite plate is completely solved. 


\section{SOUND TRANSMISSION LOSS}

The power of incident sound is defined by $[5,16]$

$$
\Pi_{i n}=\frac{1}{2} \operatorname{Re} \iint_{A} p_{i n} v_{i n}^{*} d A,
$$

where the asterisk symbol denotes complex conjugate, and the acoustic particle velocity is related to the sound pressure through $v_{\text {in }}^{*}=p_{\text {in }} /\left(\rho_{0} c_{0}\right)$, and the sound pressure in the incident field is defined by

$$
p_{i n}=j \rho_{0} \omega \Phi_{1}(x, y, 0)=j \rho_{0} \omega\left[2 I e^{-j\left(k_{x} x+k_{y} y\right)}-\sum_{m=1}^{\infty} \sum_{n=1}^{\infty} \frac{\omega}{k_{z}} \alpha_{1, m n} \varphi_{m n}(x, y)\right] .
$$

Substitution of $p_{\text {in }}$ and $v_{\text {in }}^{*}$ into (32) yields

$$
\begin{aligned}
\Pi_{i n}=\frac{\rho_{0} \omega^{2}}{2 c_{0}} \mid & 4 I^{2} \iint_{A} e^{-2 j\left(k_{x} x+k_{y} y\right)} d A-4 I \frac{\omega}{k_{z}} \sum_{m, n=1}^{\infty} \alpha_{1, m n} \iint_{A} e^{-j\left(k_{x} x+k_{y} y\right)} \varphi_{m n} d A \\
& +\frac{\omega^{2}}{k_{z}^{2}} \sum_{m, n=1}^{\infty} \sum_{k, l=1}^{\infty} \alpha_{1, m n} \alpha_{1, k l} \iint_{A} \varphi_{m n}(x, y) \varphi_{k l}(x, y) d A \mid .
\end{aligned}
$$

The transmitted sound power is defined by $[5,16]$

$$
\Pi_{t r}=\frac{1}{2} \operatorname{Re} \iint_{A} p_{t r} v_{t r}^{*} d A,
$$

where $v_{t r}^{*}=p_{t r} /\left(\rho_{0} c_{0}\right)$ is the local acoustic velocity and the sound pressure in the transmitted field is defined by

$$
p_{t r}=j \rho_{0} \omega \Phi_{3}(x, y, 0)=j \rho_{0} \frac{\omega^{2}}{k_{z}} \sum_{m=1}^{\infty} \sum_{n=1}^{\infty} \alpha_{2, m n} \varphi_{m n}(x, y)
$$

Substitution of $p_{t r}$ and $v_{t r}^{*}$ into (35) yields

$$
\Pi_{t r}=\frac{\rho_{0} \omega^{4}}{2 c_{0} k_{z}^{2}}\left|\sum_{m, n=1}^{\infty} \sum_{k, l=1}^{\infty} \alpha_{2, m n} \alpha_{2, k l} \iint_{A} \varphi_{m n}(x, y) \varphi_{k l}(x, y) d A\right| .
$$

The sound transmission loss across the laminated composite double-plate is calculated in decibel scale by

$$
S T L=10 \log _{10}\left(\frac{\Pi_{i n}}{\Pi_{t r}}\right)
$$




\section{RESULTS AND DISCUSSION}

\subsection{Validation study for finite metallic double-plate}

The present analytical approach is validated by comparing the predicted STL with the theoretical and experimental results of Carneal and Fuller in [1] as shown in Fig. 2 for a finite double-plate in the case of normal sound incidence. The double-plate considered consists of two identical aluminum plates. The geometrical dimensions of the plates are: length of plate $a=0.380 \mathrm{~m}$, width of plate $b=0.300 \mathrm{~m}$. The plate have thickness $h=$ $0.0016 \mathrm{~m}$ while the thickness of the air cavity is $H=0.048 \mathrm{~m}$. The mechanical properties of aluminum materials are: $E=70 \mathrm{GPa} ; \rho=2700 \mathrm{~kg} / \mathrm{m}^{3} ; v=0.33$. The air speed of sound, $c=343 \mathrm{~m} / \mathrm{s} ; \rho=1.21 \mathrm{~kg} / \mathrm{m}^{3}$; the amplitude of the acoustic velocity potential for the incident sound is $I=1 \mathrm{~m}^{2} / \mathrm{s}$.

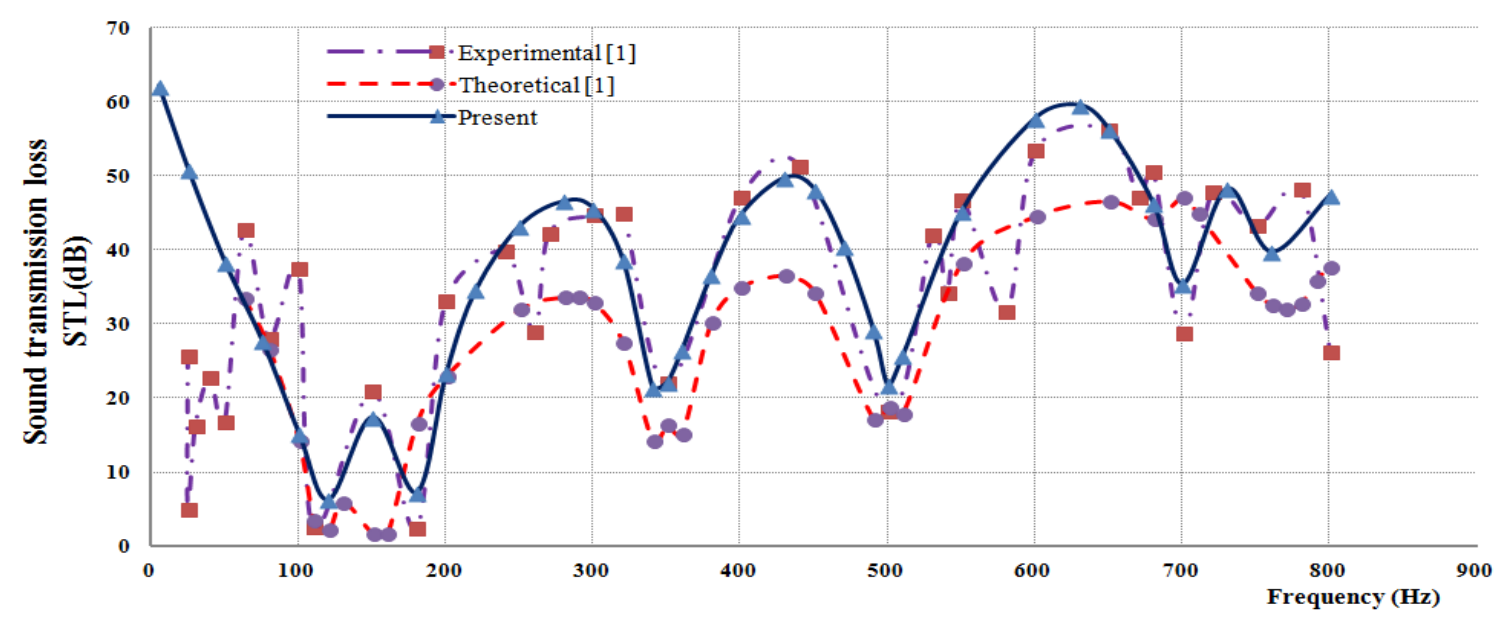

Fig. 2. Comparison of the present analytical predictions with the theoretical and experimental results of Carneal and Fuller in [1], $\left(\varphi=0^{\circ}\right)$

Fig. 2 indicates clearly that the present analytical predictions are in good agreement with the experimental measurements than the theoretical predictions of Carneal and Fuller in [1]. Notice first that the experimental results are not reliable for frequencies below $50 \mathrm{~Hz}$ where the flanking paths of the test facility play a prominent role in measurements. Second, the extra dips in the experiment curve compared with the theoretical curves associated with $240-270 \mathrm{~Hz}$ and $490-570 \mathrm{~Hz}$ modes are attributed to the imperfect normal acoustic plane wave and/or the structural flanking path, as emphasized by Carneal and Fuller [1].

\subsection{STL of a finite aluminum and of an orthotropic double-composite plates}

In this section, we have calculated the sound propagation loss through two doubleplates: a finite isotropic (aluminum) double-plate and an orthotropic laminated doublecomposite plate. Two plates have the similar geometric parameters: length of plate $a=$ $1 \mathrm{~m}$; width of plate $b=1 \mathrm{~m}$; thickness of faceplate $h=0.002 \mathrm{~m}$; thickness of air cavity $H$ $=0.08 \mathrm{~m}$. The mechanical properties of aluminum materials are presented in section 4.1 
and the composite plates are made up of graphite/epoxy with the plies being arranged in a $[0 / 90 / 0 / 90]_{s}$ pattern with mechanical properties: $E_{1}=137 \mathrm{GPa} ; E_{2}=10 \mathrm{GPa} ; \mathrm{G}_{12}=$ $5 \mathrm{GPa} ; v_{12}=0.30 ; \rho=1590 \mathrm{~kg} / \mathrm{m}^{3}$.

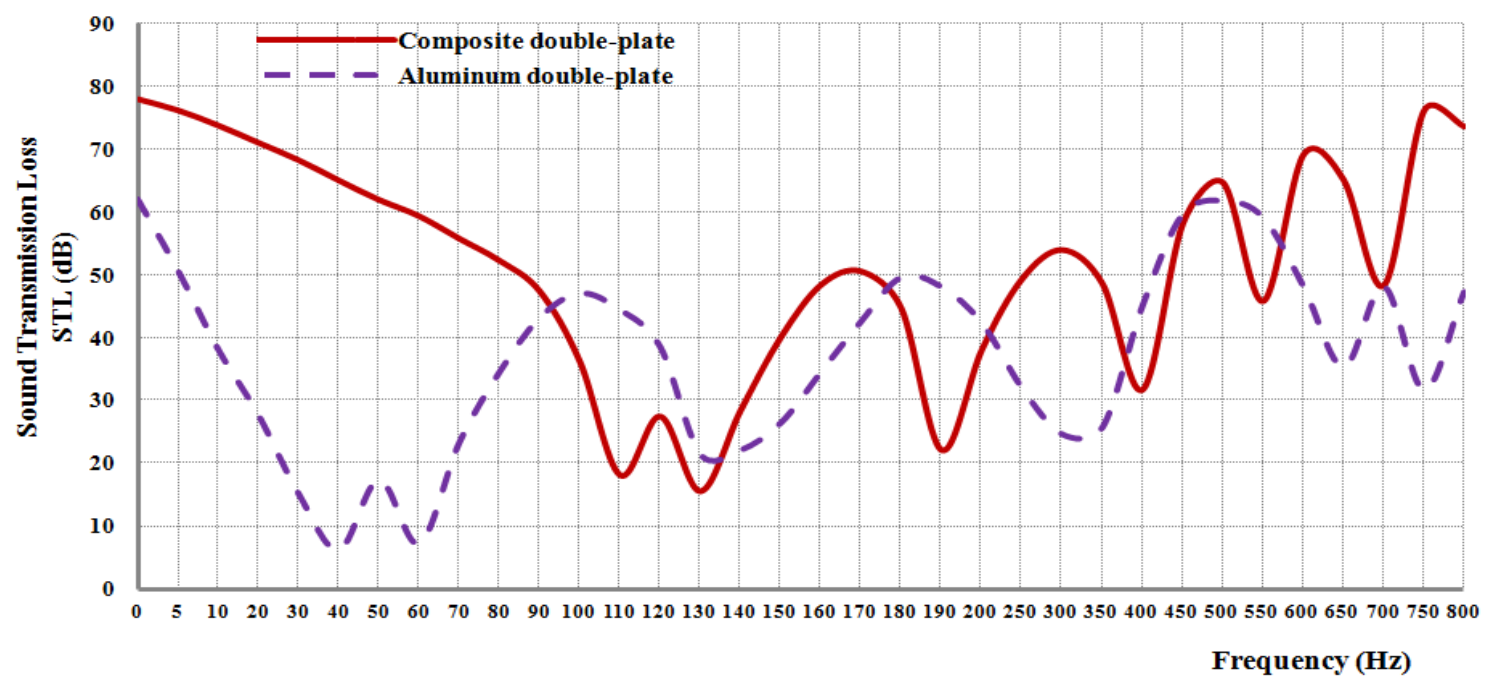

Fig. 3. STL of a finite aluminum and an orthotropic double-composite plates with incident angle $\varphi=30^{\circ}$ and azimuth angle $\theta=0^{\circ}$

As can be seen in Fig. 3 that, the dip points of the two curves are different (for the aluminum double-plates at the frequency of $40-60 \mathrm{~Hz}$, whereas for the composite doubleplate at the frequency $110-130 \mathrm{~Hz}$ ). This difference is because the dip points depend entirely on surface density of plate. When going through these frequencies, we see that the two curves have the same rules because the plate-air cavity-plate resonance plays a decisive role. At frequencies greater than $600 \mathrm{~Hz}$, when the resonance is operating synchronously, the two curves will operate according to independent rules.

\subsection{Sound transmission loss of a composite single-plate and double-plate}

We compare the STL of two finite orthotropic laminated composite plates: single composite plate with the mass density with thickness $(h)$ and double-composite plate with the same mass density with thickness $(h / 2+h / 2)$. The dimensions of two plates are: $a=b=1 \mathrm{~m}$, thickness of the single-plate $h=0.01 \mathrm{~m}$ and for the double-plate: thickness of each faceplate $h=0.005 \mathrm{~m}$, thickness of the air cavity $H=0.05 \mathrm{~m}$. The single composite plate is made of 16 plies: [0/90/0/90/90/0/90/0 $]_{s}$ whereas that, each faceplate of double-composite plate consists of 8 plies: $[0 / 90 / 0 / 90]_{s}$.

From Fig. 4, it is observed that, the curves of STL of the single-plate and doubleplate are quite similar at frequency below $200 \mathrm{~Hz}$, where the surface density of the plate is dominated. Above frequency $200 \mathrm{~Hz}$, the difference between the two curves is significant, because of the resonance phenomenon (plate-cavity-plate) of double-plate, whereas the STL of single-plate tends to follow the mass law. The STL of double plate is higher than 
that of single plate with the same effective thickness. In other words, in all the frequency ranges, the double-composite plates display superior sound insulation than the sing plate

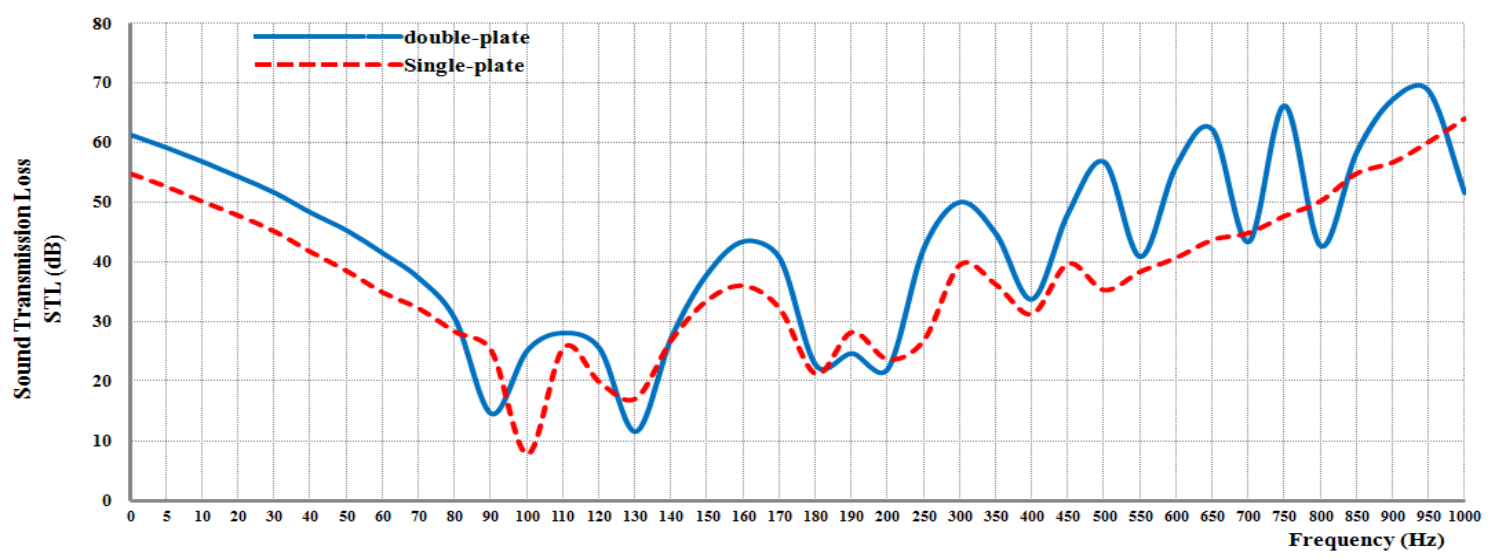

Fig. 4. Comparison of STL across an orthotropic composite single-plate and double-composite plate with incident angle $\varphi=30^{\circ}$ and azimuth angle $\theta=0^{\circ}$

In addition, by neglecting the appearance of air cavity $(H=0)$, we observed that the curve of STL of double-composite plate is almost coincided with that of single composite plate for all range of frequency considered. Once again, that affirmed the reliability of our results for STL across a clamped double-composite plate with an air cavity.

\subsection{Influence of several parameter on sound transmission loss}

In this section, the effects of incident angles plate thickness and the air cavity thickness on STL of clamped double-composite plate are discussed.

\section{Influence of incident angles}

The effect of sound incident angles (elevation angle and azimuth angle) on the sound insulation behavior of a finite clamped orthotropic laminated composite double-plate is considered. The dimensions of the plates are chosen as follows: length of plate $a=1$ $\mathrm{m}$, width of plate $b=1 \mathrm{~m}$. The plate have thickness $h=0.005 \mathrm{~m}$, the thickness of the air cavity $H=0.08 \mathrm{~m}$. The double-plate consists of two identical orthotropic laminated composite faceplates. Laminate configuration of bottom and upper plate is $[0 / 90 / 0 / 90]_{s}$. The mechanical properties of composite material, the air speed of sound, the air density and the initial amplitude of the incident sound are presented in the above sections.

Fig. 5 shows considerable influence of the incident elevation angle $\varphi$ (with azimuth angle fixed at $\theta=0^{\circ}$ ) on the STL of the clamped finite double-plate: the incident sound waves with large elevation angles are easier to transmit through the double-plate than those with smaller elevation angles. For the case studied, the STL values decrease with increasing elevation angle for frequencies below about $90 \mathrm{~Hz}$, while for frequencies above this value the overall trend is similar apart from the complicated system modal behavior.

The sound insulation properties of the clamped finite double-plate partition for selected incident azimuth angles $\left(\theta=15^{\circ}, 30^{\circ}, 45^{\circ}, 60^{\circ}\right)$ are compared in Fig. 6 , (with the 


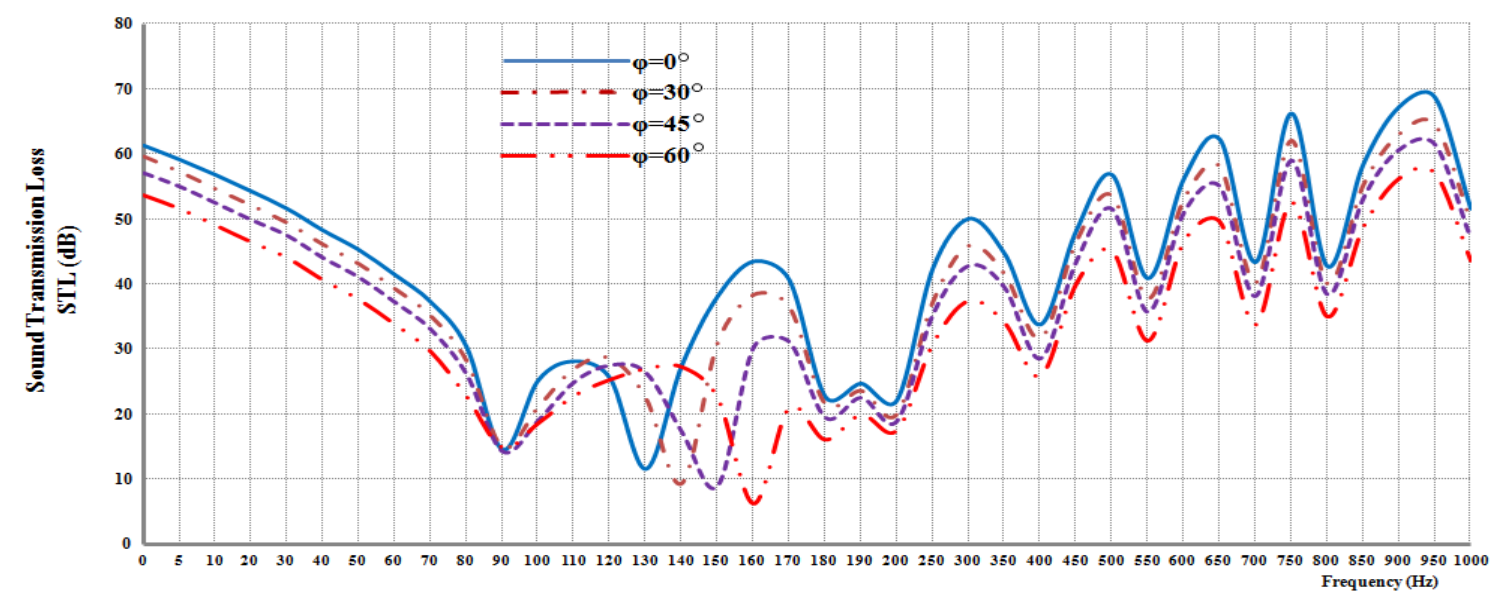

Fig. 5. Influence of incident angle on STL of clamped composite double-plate excited by incident sound having different elevation angles $\varphi$ (azimuth angle fixed at $\theta=0^{\circ}$ )

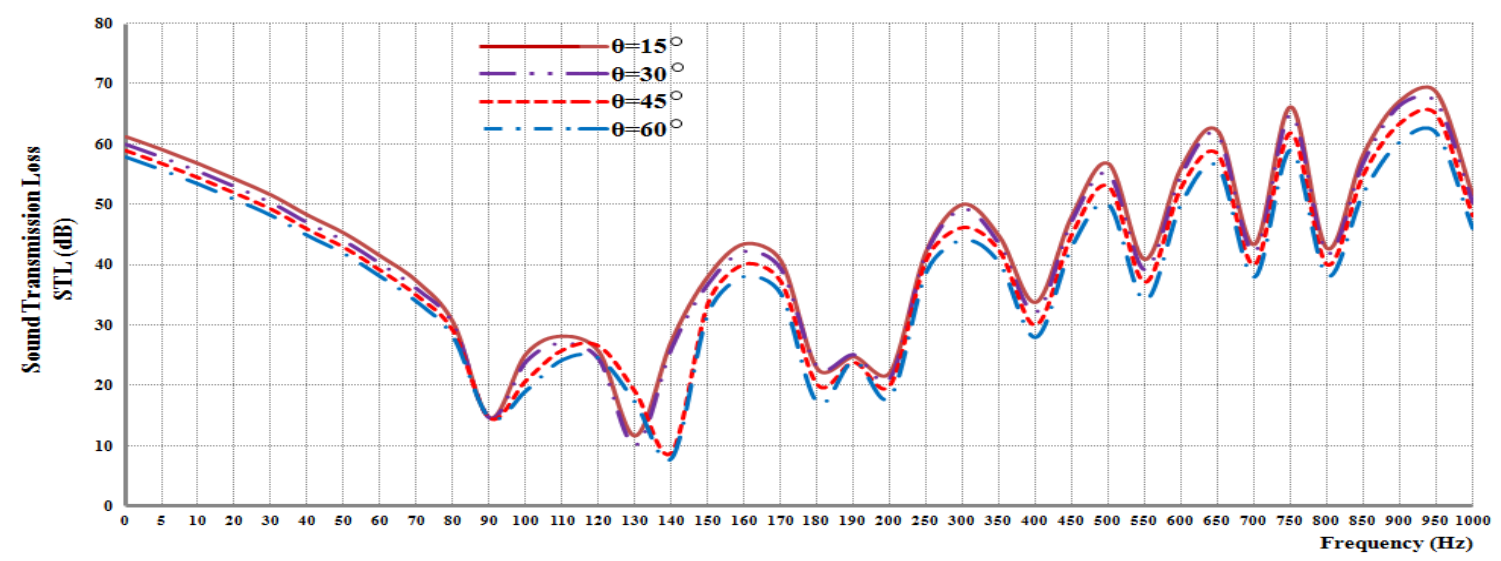

Fig. 6. Influence of azimuth angle on STL of clamped double-plate excited by incident sound having different azimuth angles $\theta$ (elevation angle fixed at $\varphi=30^{\circ}$ )

elevation angle fixed at $\varphi=30^{\circ}$ ). For frequencies below about $140 \mathrm{~Hz}$, the STL curve varies very little for four cases. At high frequencies, the change of the four curves is more obvious. Therefore, it may be concluded that the incident azimuth angle has negligible influence on the STL behavior of clamped finite double-plate.

Influence of faceplate thickness $(h)$

To quantify the influence of faceplate thickness, the STL versus frequency curve is presented in Fig. 7 for a finite double-plate. Three values of plate thickness are chosen: $h$ $=0.002,0.005$ and $0.01 \mathrm{~m}$. The dimensions of the plate are: $a \times b=1 \mathrm{~m} \times 1 \mathrm{~m}$ and the thickness of the air cavity is fixed at $H=0.08 \mathrm{~m}$. The material properties are the same as in the subsection 3.1.

According to Fig. 7, the STL value increases sharply when increasing the thickness of the plate. Effect of thickness of faceplate for STL is particularly strong at frequencies 


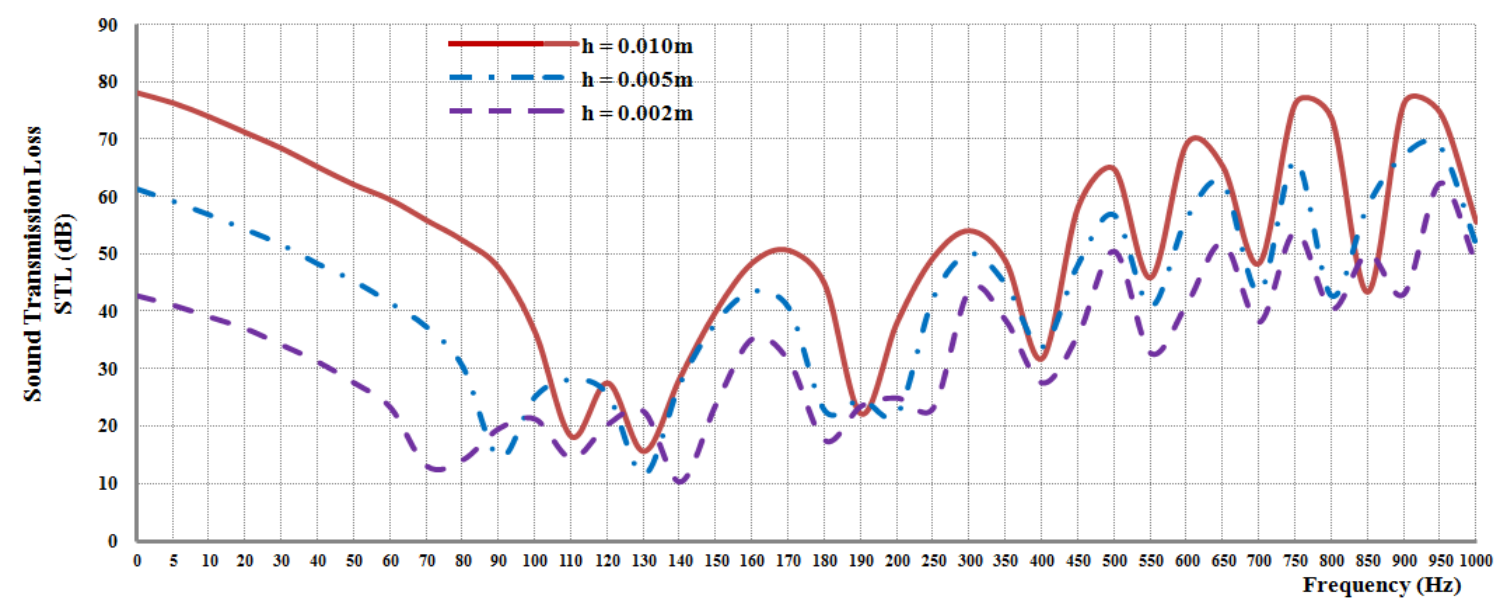

Fig. 7. Effects of plate thickness on STL of clamped double-composite plate with enclosed air cavity. Three different plate thicknesses $(h=0.002,0.005$ and $0.01 \mathrm{~m})$ were considered

lower than $100 \mathrm{~Hz}$. This is a very important region when designing finite dual sound insulation plates in practice. At higher frequencies, when peaks and poles appear in this mode, this is attributed to the strong interaction of individual plate behavior with the overall system performance for finite system. The position of double-plate resonances in Fig. 7 moves to higher frequencies when the thickness of the faceplate is increased.

\section{Influence of air cavity thickness $(H)$}

To demonstrate the influence of air cavity thickness on STL, the STLs are calculated for a finite double-composite plate with selected values of air cavity thickness: $H=0.02$, 0.04 , and $0.08 \mathrm{~m} ; h_{1}=h_{2}=0.01 \mathrm{~m}$, as shown in Fig. 8 . The other geometrical and material parameters are the same as section 4.2 and normal sound excitation is imposed.

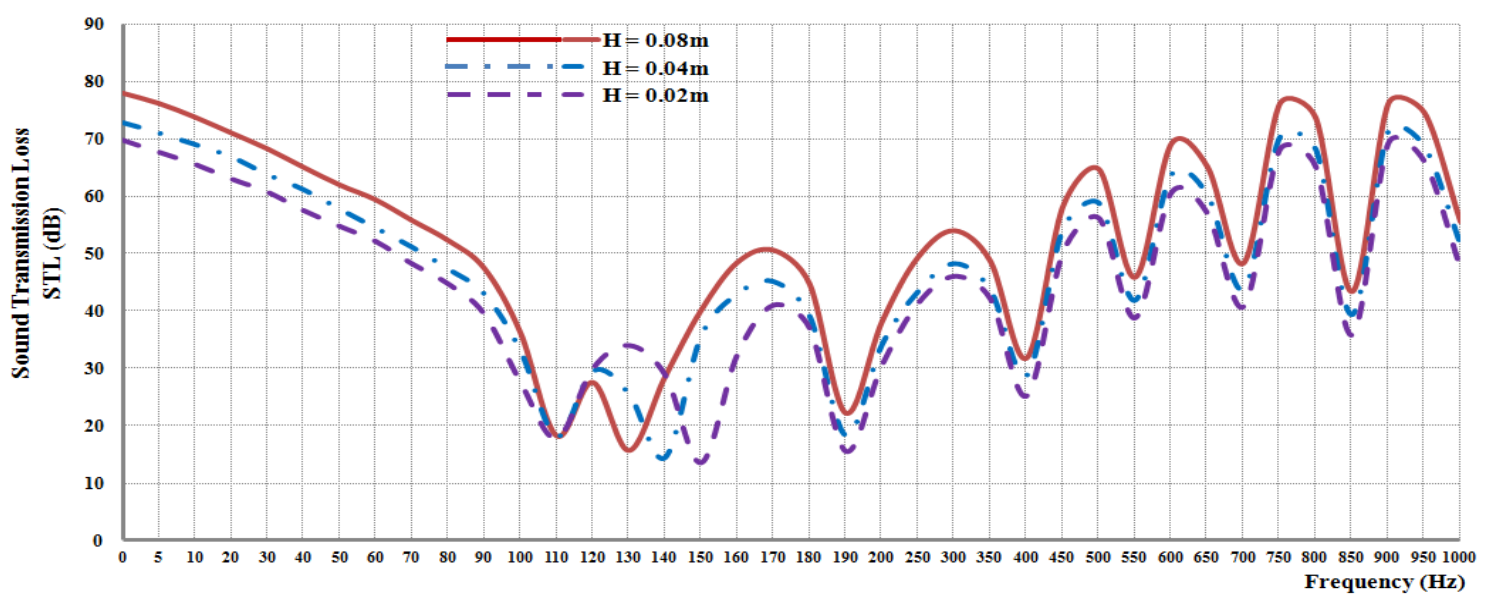

Fig. 8. Effects of air cavity thickness on STL of clamped double-composite plate with enclosed air cavity. Three different air cavity thicknesses (i.e., $0.02,0.04$, and $0.08 \mathrm{~m}$ ) were considered 
As can be seen in Fig. 8, the first dip position does not depend on the air cavity thickness because it completely depends on the surface density of the plate. However, the second dip position changes drastically when the air cavity thickness increases (moving toward the lower frequency, Fig. 8) due to the fact that the plate-cavity-plate resonance plays a major role in this case. Their remaining positions are almost unchanged because the plate-cavity-plate is operating synchronously. Therefore, by tailoring the thickness of air cavity, it is possible to design finite double-plate partitions with better sound insulation properties over a wide frequency range.

\section{CONCLUSIONS}

An analytical approach has been developed to study the vibroacoustic behavior and the sound transmission loss across a clamped orthotropic laminated composite doubleplate with enclosed air cavity. We get some of the following conclusions:

- The theoretical predictions are in good agreement with existing experimental results.

- The sound insulation capacity of double-composite plates is better than that of the aluminum double-plates when they have the same geometric parameters.

- The double-composite plate has better sound insulation than the single-composite plate when they have the same geometric parameters and the same mechanical properties.

- When the incident angle of the incident sound waves increases, the sound transmission loss decreases. On the other hand, the incident azimuth angle has negligible influence on the STL of a finite clamped double-composite plate.

- The influence of plate thickness on STL is particularly strong for the finite systems at low frequencies, which is useful when designing clamped sound-proof doublecomposite plates.

- As the thickness of the air cavity increases, the sound insulation capacity of the double-composite plate also increases but is not as strong as changing the thickness of the plate.

\section{ACKNOWLEDGEMENTS}

This research is funded by Vietnam National Foundation for Science and Technology Development (NAFOSTED) under grant number: 107.02-2018.07.

\section{REFERENCES}

[1] J. P. Carneal and C. R. Fuller. An analytical and experimental investigation of active structural acoustic control of noise transmission through double panel systems. Journal of Sound and Vibration, 272, (3-5), (2004), pp. 749-771. https://doi.org/10.1016/s0022-460x(03)00418-8.

[2] S. J. Pietrzko and Q. Mao. New results in active and passive control of sound transmission through double wall structures. Aerospace Science and Technology, 12, (1), (2008), pp. 42-53. https://doi.org/10.1016/j.ast.2007.10.006.

[3] J. D. Quirt. Sound transmission through windows I. Single and double glazing. The Journal of the Acoustical Society of America, 72, (3), (1982), pp. 834-844. https://doi.org/10.1121/1.388263. 
[4] J. D. Quirt. Sound transmission through windows II. Double and triple glazing. The Journal of the Acoustical Society of America, 74, (2), (1983), pp. 534-542. https://doi.org/10.1121/1.389819.

[5] T. Lu and F. Xin. Vibro-acoustics of lightweight sandwich structures. Springer, (2014).

[6] G. Maidanik. Response of ribbed panels to reverberant acoustic fields. The Journal of the Acoustical Society of America, 34, (6), (1962), pp. 809-826. https://doi.org/10.1121/1.1918200.

[7] M. Ruzzene. Vibration and sound radiation of sandwich beams with honeycomb truss core. Journal of Sound and Vibration, 277, (4-5), (2004), pp. 741-763. https://doi.org/10.1016/j.jsv.2003.09.026.

[8] A. London. Transmission of reverberant sound through double walls. The Journal of the Acoustical Society of America, 22, (2), (1950), pp. 270-279. https://doi.org/10.1121/1.1906601.

[9] W. Kropp and E. Rebillard. On the air-borne sound insulation of double wall constructions. Acta Acustica United with Acustica, 85, (5), (1999), pp. 707-720.

[10] M. Villot, C. Guigou, and L. Gagliardini. Predicting the acoustical radiation of finite size multi-layered structures by applying spatial windowing on infinite structures. Journal of Sound and Vibration, 245, (3), (2001), pp. 433-455. https://doi.org/10.1006/jsvi.2001.3592.

[11] J. D. Chazot and J. L. Guyader. Prediction of transmission loss of double panels with a patchmobility method. The Journal of the Acoustical Society of America, 121, (1), (2007), pp. 267-278. https://doi.org/10.1121/1.2395920.

[12] C. Bao and J. Pan. Experimental study of different approaches for active control of sound transmission through double walls. The Journal of the Acoustical Society of America, 102, (3), (1997), pp. 1664-1670. https://doi.org/10.1121/1.420105.

[13] B. Brouard, D. Lafarge, and J. F. Allard. A general method of modelling sound propagation in layered media. Journal of Sound and Vibration, 183, (1), (1995), pp. 129-142. https://doi.org/10.1006/jsvi.1995.0243.

[14] W. Lauriks, P. Mees, and J. F. Allard. The acoustic transmission through layered systems. Journal of Sound and Vibration, 155, (1), (1992), pp. 125-132. https://doi.org/10.1016/0022460x(92)90650-m.

[15] W. Larbi, J. F. Deü, and R. Ohayon. Vibroacoustic analysis of double-wall sandwich panels with viscoelastic core. Computers \& Structures, 174, (2016), pp. 92-103. https://doi.org/10.1016/j.compstruc.2015.09.012.

[16] R. Panneton and N. Atalla. Numerical prediction of sound transmission through finite multilayer systems with poroelastic materials. The Journal of the Acoustical Society of America, 100, (1), (1996), pp. 346-354. https://doi.org/10.1121/1.415956.

[17] S. Chonan and Y. Kugo. Acoustic design of a three-layered plate with high sound interception. The Journal of the Acoustical Society of America, 89, (2), (1991), pp. 792-798. https://doi.org/10.1121/1.1894639.

[18] H.-J. Kang, J.-G. Ih, J.-S. Kim, and H.-S. Kim. Prediction of sound transmission loss through multilayered panels by using Gaussian distribution of directional incident energy. The Journal of the Acoustical Society of America, 107, (3), (2000), pp. 1413-1420. https://doi.org/10.1121/1.428428.

[19] J. S. Bolton, N.-M. Shiau, and Y. J. Kang. Sound transmission through multi-panel structures lined with elastic porous materials. Journal of Sound and Vibration, 191, (3), (1996), pp. 317-347. https://doi.org/10.1006/jsvi.1996.0125.

[20] F. G. Leppington, E. G. Broadbent, and G. F. Butler. Transmission of sound through a pair of rectangular elastic plates. IMA Journal of Applied Mathematics, 71, (6), (2006), pp. 940-955. https://doi.org/10.1093/imamat/hx1017. 
[21] T. I. Thinh and P. N. Thanh. Vibro-acoustic response of an orthotropic composite laminated plate. In Proceedings of National Conference on Mechanics, Hanoi, Vietnam, (2017). pp. 11421150.

[22] T. I. Thinh and P. N. Thanh. Vibro-acoustic response of a finite clamped laminated composite plate. In Proceedings of International Conference on Engineering and Research Application, Thai Nguyen, Vietnam, (2019). pp. 589-600, https:/ /doi.org/10.1007/978-3-030-04792-4_76.

[23] A. Pellicier and N. Trompette. A review of analytical methods, based on the wave approach, to compute partitions transmission loss. Applied Acoustics, 68, (10), (2007), pp. 1192-1212. https://doi.org/10.1016/j.apacoust.2006.06.010.

[24] K. D. Frampton. The effect of flow-induced coupling on sound radiation from convected fluid loaded plates. The Journal of the Acoustical Society of America, 117, (3), (2005), pp. 11291137. https://doi.org/10.1121/1.1852894.

[25] V. Q. Hien, T. I. Thinh, and N. M. Cuong. Free vibration analysis of joined composite conical-cylindrical-conical shells containing fluid. Vietnam Journal of Mechanics, 38, (4), (2016), pp. 249-265. https://doi.org/10.15625/0866-7136/6954.

[26] A. W. Leissa. Vibration of plates. Acoustical Society of America, New York, (1993).

[27] M. S. Howe and P. L. Shah. Influence of mean flow on boundary layer generated interior noise. The Journal of the Acoustical Society of America, 99, (6), (1996), pp. 3401-3411. https://doi.org/10.1121/1.414988. 